\title{
Spall fracture of copper under loading by shock waves with duration less 1 microsecond
}

\author{
V.I. Skokov ${ }^{1, a}$, O.N. Ignatova ${ }^{1}$, A.N. Malyshev ${ }^{1}$, A.M. Podurets ${ }^{1}$, V.A. Raevsky ${ }^{1}$, M.I. Tkachenko ${ }^{1}$, \\ and M.A. Zocher ${ }^{2}$ \\ 1 FSUE "RFNC - VNIIEF", Sarov, Russia \\ 2 Los Alamos National Laboratory, Los Alamos NM 87545, USA
}

\begin{abstract}
The paper presents results of experimental researches on spall fracture of polycrystalline copper when loading by shock waves with intensity of $20-33 \mathrm{GPa}$ and duration $<1 \mu \mathrm{s}$. It is shown that decrease of copper spall strength is observed behind SW front at SW intensity of $\sim 33 \mathrm{GPa}$ (above threshold of formation of heterogeneous deformation bands). When reducing SW intensity to $27 \mathrm{GPa}$, spall strength is restored. Duration of the softened copper state does not exceed $\sim 0.5 \mu \mathrm{s}$. The method for spall strength evaluation is based on measurement of maximum damage in post-test samples.
\end{abstract}

\section{Introduction}

In work [1], significant reduction of spall strength of polycrystalline copper was experimentally recorded when loading by planar shock wave having intensity of $\sim 33 \mathrm{GPa}$ and duration less $1 \mu \mathrm{s}$. During development of setup for these tests, the authors were basing on the following main statements:

- first, it was assumed that the phenomenon of temporal softening has a general, universal character under shock-wave loading of polycrystalline metals. In the other words, under certain conditions, it should be manifested in both shear strength and spall strength. The problem of experimental revealing of this phenomenon concerning metal resistance against tensile stresses becomes urgent due to the fact that this phenomenon is experimentally confirmed [2,3] for shear strength;

- second, when developing the experimental setup for tests aimed to reveal decrease of metal spall strength behind SW front, it is required to meet some basic conditions. It is known that the process of formation of a complicated heterogeneous structure of deformed metal is threshold in pressure and strain rate. For polycrystalline copper with grain size of $110 \mu \mathrm{m}$, critical pressure in shock wave is $\sim 27-28 \mathrm{GPa}$, strain rate is $\sim 10^{7} \mathrm{~s}^{-1}$ [4]. On the other hand, since duration of existence of softened state of metal behind SW front is limited due to temperature heterogeneity, and it is equal to $\sim 0.3-0.5 \mu$ s according to estimations [4], metal should be loaded by pulse of tensile stresses for recording the phenomenon of spall strength reduction after SW front passage also during this time range.

Therefore, according to the hypothesis of short-time softening, it follows that it is possible to expect a significant reduction of metal spall strength when loading polycrystalline copper with grain size of $110 \mu \mathrm{m}$ by shock wave having intensity of $\sim 33 \mathrm{GPa}$ and by rarefaction wave, which goes after its front in $0.3-0.5 \mu \mathrm{s}$. The main goal of the researches was experimental recording of this fact. The recording method is based on measurement of value of the maximum damage in sample, which is subjected to shock-wave loading for less than $1 \mu$ s followed by unloading.

\footnotetext{
a e-mail: root@gdd.vniief.ru
}

This is an Open Access article distributed under the terms of the Creative Commons Attribution-Noncommercial License 3.0, which permits unrestricted use, distribution, and reproduction in any noncommercial medium, provided the original work is properly cited. 


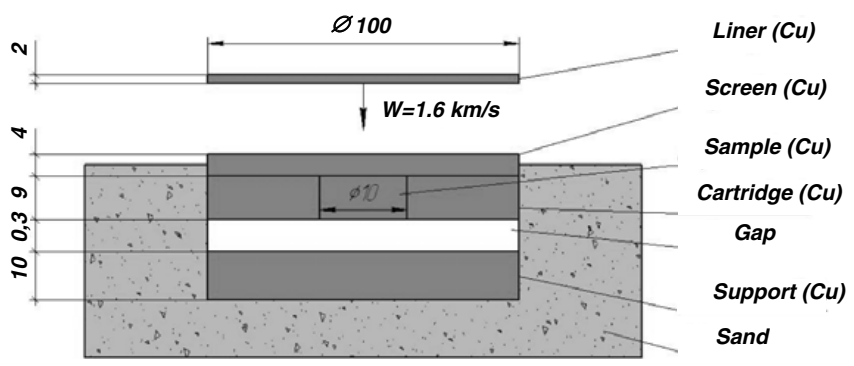

Fig. 1. Scheme of experimental device.

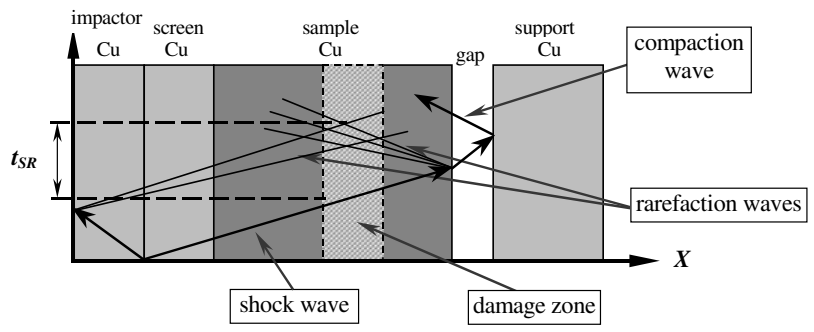

Fig. 2. Scheme of wave processes in experimental device.

Table 1. Calculated values of $t_{S R}$ for conducted tests.

\begin{tabular}{|l|c|c|c|c|}
\hline Number of device & 1 & 2 & 3 & 4 \\
\hline Reduction scale & $1: 1$ & $1: 2$ & $1: 4$ & $1: 8$ \\
\hline$t_{S R}, \mu$ s & 2 & 1 & 0.5 & $0.3 \div 0.25$ \\
\hline
\end{tabular}

\section{Experimental setup}

To provide the specified conditions in copper samples for high-intensive loading followed by effect of pulse of tensile stresses with time delay $t_{S R} \sim 0.3-1.0 \mu \mathrm{s}$, an experimental setup is suggested. It is presented in Fig. 1. For the researches, we used samples made of M1 type copper with the initial grain size of $110 \mu \mathrm{s}$. The samples were annealed for one hour at $550{ }^{\circ} \mathrm{C}$. Then they were cooled down in air. Shock-wave loading was performed by the impact method. Velocities of the copper liner were $\sim 1600$ and $\sim 1000 \mathrm{~m} / \mathrm{s}$. In the main part of the tests, the liner was accelerated by HE in the regime of sliding detonation. Copper sample, which was pressed into a copper cartridge, was covered with a copper screen. Pulse of tensile stresses was formed in the sample when it was unloaded into a gap. To form a shock wave of compaction, a copper substrate was mounted. However, as it is shown in [1], there is an area in the sample, where its influence on damage value $\omega$ can be neglected.

The experimental device was manufactured in four variants, which differed in longitudinal sizes. The basic variant of the assembly (scale of 1) is presented in Fig. 1. The remaining three variants of the devices were produced by 2 (scale of 1:2), 4 (scale of 1:4), 8 (scale of 1:8) times reducing the longitudinal sizes of the basic variant. The liner velocity was kept constant.

Scheme of the basic wave processes, which occur in the device, is presented in Fig. 2; calculated profiles $\sigma_{X}(X)$ in the sample for four devices at the time of SW front arrival to its free boundary are presented in Fig. 3; calculated profiles $\sigma_{x}(t)$ of tensile stresses in the plane of their maximum value are given in Fig. 4; expected (calculated) maximum damage of the samples is presented in Fig. 5; calculated values of time of rarefaction wave arrival regarding to the $\mathrm{SW}$ front $\left(t_{S R}\right)$ are given in Table 1.

The calculations were performed by the one-dimensional technique UP [5] with use of the relaxation model of strength and the model of copper spall strength of the NAG type [6]. According to the existing knowledge, $\sim 1.3$ times reduction of damage $\omega$ was expected as the assembly scale was 


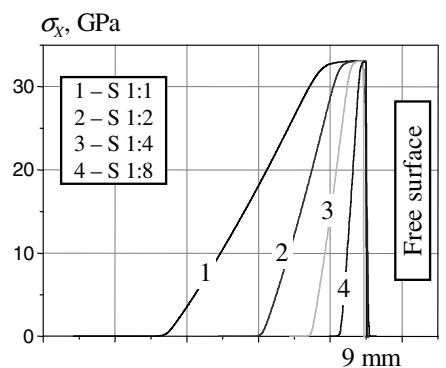

Fig. 3. Calculated profiles $\sigma_{X}(X)$ in sample at time of SW front arrival to its free boundary for four devices at impactor velocity of $1600 \mathrm{~m} / \mathrm{s}$.

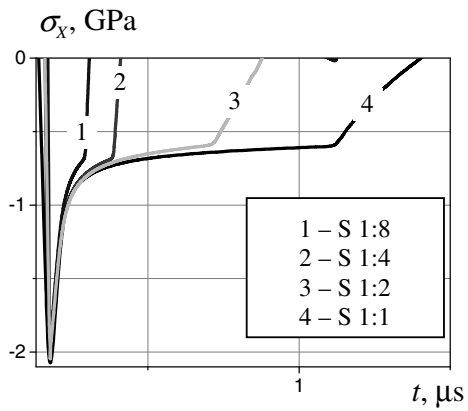

Fig. 4. Calculated profiles $\sigma_{X}(t)$ of tensile stresses in plane of their maximum value.

8 times reduced (see Fig. 5) [7,8]. Also it should be noted that, according to the data from Table 1, time $\underline{t}_{S R}$, is comparable to the time of short-time loss of strength in the device with the scale of 1:4; and the sample should be in the softened state for sure in the device having the scale of 1:8 at time of rarefaction wave arrival.

\section{Experimental results}

The tested samples were investigated with use of metallographic microscope METAM LV-31. Damage was determined by the following method. Measuring bar with length of $1.6 \mathrm{~mm}$ was placed in the required area of microsection in field of view of the microscope at $100^{\times}$magnification. We measured the relation of total length of pores, which were on the measuring bar, to its length. This relation (in percent) was considered as the damage value.

\subsection{Tests with SW intensities of $32-33 \mathrm{GPa}$ and $20 \mathrm{GPa}$}

Bands of localized deformation can be seen in grains along all sample section. Volume of grains, which were covered by bands of localized deformation, was $\sim 30-40 \%$ in the samples produced in the devices with the scale of $1: 1$; it was $\sim 60-70 \%$ with scales of 1:2, 1:4, and 1:8. Width of shear bands was $l \sim 1-2 \mu \mathrm{m}$, and their period was $L \sim 5-10 \mu \mathrm{m}$. Compaction traces were observed in all samples.

For all samples, results of damage measurement are presented in Fig. 6. Also it presents the calculated dependence $\omega\left(t_{S R}\right)$.

The experimental dependence $\omega\left(t_{S R}\right)$ from Fig. 6 points to the fact that abrupt change of its behavior character can be observed at $t_{S R} \sim 0.5 \mu \mathrm{s}$, namely, damage $\sim 2$ times grows as compared to the expected value. Reduction of $t_{S R}$ to $0.25 \mu$ s causes further growth of damage up to the value of $\sim 50 \%$. These results testify to decrease of resistance against tensile stresses of copper (spall strength) directly behind the SW front under conditions of heterogeneous deformation. 


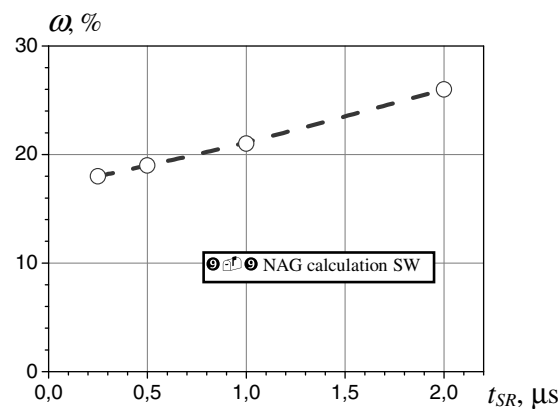

Fig. 5. Expected values of damage of tested samples depending on assembly scale.

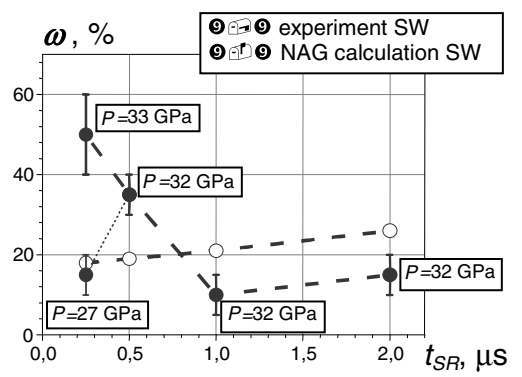

Fig. 6. Experimental and calculated dependences of maximum damage of sample on time of rarefaction wave arrival.

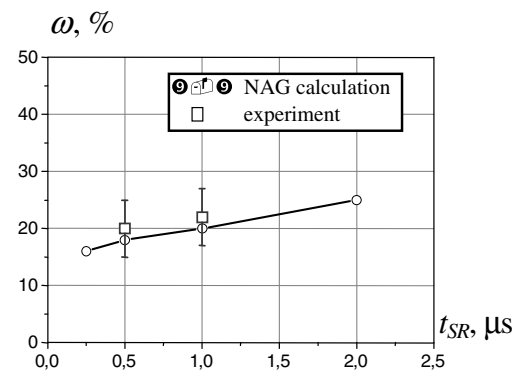

Fig. 7. Damage versus $t_{S R}$ at $\sigma_{X}=20 \mathrm{GPa}$.

The supposition that presence of deformation packages of twins is the necessary condition of softening was experimentally checked. The test, which was performed at $t_{S R}=0.25 \mu \mathrm{s}$, but at SW intensity of $27 \mathrm{GPa}$ (lower than threshold of formation of localized deformation bands), as compared to the test at $\sigma_{X}=33 \mathrm{GPa}$, gave immediately threefold reduction of damage (see Fig. 6), i.e. no any softening is observed, and the obtained result can be described by existing models.

Besides, to justify the conclusion on direct association of the heterogeneous character of deformation and reduction of spall strength directly behind $\mathrm{SW}$ front, tests were performed at impactor velocity of $\sim 1000 \mathrm{~m} / \mathrm{s}\left(\sigma_{X}=20 \mathrm{GPa}\right)$ using devices with the scale of $1: 2\left(t_{S R}=1.0 \mu \mathrm{s}\right)$ and $1: 4\left(t_{S R}=0.5 \mu \mathrm{s}\right)$. These points are depicted in the graph, which is presented in Fig. 7. Also the figure presents the calculated dependence $\omega\left(t_{S R}\right)$, which was determined by the NAG model. It follows from comparison of these results that there is no any phenomenon of copper spall strength reduction observed, if there is no formation of localized deformation bands. 


\section{Conclusion}

1. The performed researches show that copper spall strength reduction is observed in polycrystalline copper having grain size of $\sim 110 \mu \mathrm{m}$ under shock-wave loading with intensity of $\sim 33$ GPa behind SW front.

2. Duration of existence of softened state behind SW front is $\sim 0.5 \mu$ s or less.

3. Short-time softening of copper is caused by formation of heterogeneous deformation bands.

The work was supported by Los Alamos National Laboratory and Scientific School NSh-1307.2008.1.

\section{References}

1. V.I. Skokov, O.N. Ignatova, A.N. Malyshev, A.M. Podurets, V.A. Raevsky, M.A. Zocher, Short-time softening and spall strength of materials (Zababakhin's Scientific Talks, 2008)

2. L.C. Chhabildas, J.R. Asay, Dynamic yield strength and spall strength measurement under quasiisentropic loading (Conf. Shock Wave and High Strain Phenomena in Materials, New York, p. 947, 1992)

3. A.I. Lebedev, O.N. Aprelkov, V.A. Arinin et al, Perturbation method for study of shear strength of materials at pressures up to $300 \mathrm{GPa}$ (In - Shock Compression of Condensed Matter-2005, Melville, New York, p. 745-748, 2006)

4. V.A. Raevsky, M.A. Podurets, V.G. Khanzhin et al, Twin structures in copper after shock and shockless high-rate loading (IX Khariton's Topical Scientific Readings, Extreme states of substance, Detonation, Shock waves, Sarov, 2007)

5. N.F. Gavrilov, G.G. Ivanova, V.I. Selin, V.N. Sofronov, UP-OK program for solution of onedimensional problems of solid medium mechanics in one-dimensional complex, VANT, issue 3(11), p. 11-14 (1982)

6. O.N. Ignatova, V.A. Raevsky, O.A. Tyupanova, V.I. Skokov et al, Peculiarities of damage nucleation stage at intensive loading of copper (Proceedings of International Conference "the VII Khariton's Topical Scientific Readings", Sarov, 2005)

7. A.G. Ivanov, V.N. Mineev, On scale effects during destruction (Physics of Combustion and Explosion. \# 5, p. 70-95, 1979)

8. V.A. Ogorodnikov, A.G. Ivanov, V.I. Luchinin et al, On nature of scale effect at high-velocity destruction (spall) (Physics of Combustion and Explosion, \# 6, p. 88-93, 1993) 